Corresponding authors: dg2875@cumc.columbia.edu, vandana.shashi@duke.edu

(C) 2015 Petrovski et al. This article is distributed under the terms of the Creative Commons Attribution-NonCommercial License, which permits reuse and redistribution, except for commercial purposes, provided that the original author and source are credited.

Ontology terms: drooling, gait imbalance, neck muscle weakness, neurodegeneration, seesaw nystagmus, upper motor neuron abnormality

Published by Cold Spring Harbor Laboratory Press

doi: $10.1101 /$ mcs.a000257

\section{Exome sequencing results in successful riboflavin treatment of a rapidly progressive neurological condition}

\author{
Slavé Petrovski, ${ }^{1,2,9}$ Vandana Shashi, ${ }^{3,9}$ Steven Petrou, ${ }^{4}$ Kelly Schoch, ${ }^{3}$ Keisha \\ Melodi McSweeney, ${ }^{1}$ Ryan S. Dhindsa, ${ }^{1}$ Brian Krueger, ${ }^{1}$ Rebecca Crimian, ${ }^{3}$ \\ Laura E. Case, ${ }^{5}$ Roha Khalid, ${ }^{6}$ Maysantoine A. El-Dairi, ${ }^{7}$ Yong-Hui Jiang, ${ }^{3,8}$ \\ Mohamad A. Mikati, ${ }^{6}$ and David B. Goldstein ${ }^{1}$ \\ ${ }^{1}$ Institute for Genomic Medicine, Columbia University, New York, New York 10032, USA; ${ }^{2}$ Department of \\ Medicine, The University of Melbourne, Austin Health and Royal Melbourne Hospital, Melbourne, 3050 \\ Victoria, Australia; ${ }^{3}$ Department of Pediatrics, Division of Genetics, Duke University School of Medicine, \\ Durham, North Carolina 27710, USA; ${ }^{4}$ Ion Channels and Disease Group, Epilepsy Division, Florey Institute of \\ Neuroscience and Mental Health, Parkville, Victoria 3052, Australia; ${ }^{5}$ Division of Physical Therapy, Department \\ of Community and Family Medicine, Duke University Medical Center, Durham, North Carolina 27710, USA; \\ ${ }^{6}$ Department of Pediatrics, Division of Neurology, Duke University School of Medicine, Durham, North \\ Carolina 27710, USA; ${ }^{7}$ Department of Ophthalmology, Duke University School of Medicine, Durham, North \\ Carolina 27710, USA; ${ }^{8}$ Department of Neurobiology, Duke University, Durham, North Carolina 27710, USA
}

Abstract Genetically targeted therapies for rare Mendelian conditions are improving patient outcomes. Here, we present the case of a 20 -mo-old female suffering from a rapidly progressing neurological disorder. Although diagnosed initially with a possible autoimmune condition, analysis of the child's exome resulted in a diagnosis of BrownVialetto-Van Laere syndrome 2 (BVLS2). This new diagnosis led to a change in the therapy plan from steroids and precautionary chemotherapy to high-dose riboflavin. Improvements were reported quickly, including in motor strength after $1 \mathrm{mo}$. In this case, the correct diagnosis and appropriate treatment would have been unlikely in the absence of exome sequencing and careful interpretation. This experience adds to a growing list of examples that emphasize the importance of early genome-wide diagnostics.

\section{INTRODUCTION}

Approximately 90 patients have been reported with Brown-Vialetto-Van Laere syndrome (BVVLS), inclusive of those with mutations in SLC52A2 and SLC52A3 (Bosch et al. 2012). SLC52A3 mutations causing BVVLS1 disease are hypothesized to disrupt the riboflavin transporter (RFVT3) that is responsible for riboflavin uptake from food into serum (Haack et al. 2012). Consistent with that role, RFVT3 deficiency is often detected in patients through reduced riboflavin or flavin adenine dinucleotide (FAD) plasma levels. However, the gene disrupted among patient with BVVLS2, SLC52A2, encodes the transmembrane riboflavin transporter (RFVT2) hypothesized to be responsible for riboflavin uptake from the serum into the target cells (Haack et al. 2012). Consistent with this hypothesis, children with SLC52A2 mutations (BVVLS2 disease) have been described to have flavin adenine dinucleotide (FAD) plasma levels within the normal range (Haack et al. 2012) before riboflavin supplementation. For this reason, BVVLS2 will be consistently missed in current routine biochemical

\footnotetext{
${ }^{9}$ These authors contributed equally to this work.
} 
COLD SPRING HARBOR Molecular Case Studies
Exome sequencing guides appropriate patient care screening. Guided by the underlying disruption of riboflavin transporters, Foley et al. (2014) reported 16 patients with SLC52A2 mutations who were treated with high-dose riboflavin therapy. All patients tolerated the therapy well with no evidence of toxicity. Critical to patient care, these data also showed that the sooner the riboflavin supplementation is initiated in patients with riboflavin transporter deficiency, the better the prognosis (Foley et al. 2014).

There is increasing evidence that exome sequencing provides a substantial clinical benefit in a minority of patients with undiagnosed or unresolved genetic conditions. Here, we present a striking example in which the early exome sequencing of a child with a devastating undiagnosed condition resulted directly in a transformative change in the patient's management that would not have otherwise been achieved.

\section{RESULTS}

\section{Clinical Presentation and Family History}

At the age of $15 \mathrm{mo}$, with previously normal developmental milestones and no pertinent family medical history, a young girl was evaluated after an abrupt onset of vertical nystagmus (Human Phenotype Ontology term [Köhler et al. 2014]: HP:0010544). Contrast-enhanced cranial magnetic resonance imaging was normal.

At age $16 \mathrm{mo}$, an ophthalmic examination showed subtle pallor of both optic nerves. An electroretinogram showed $20 \%-50 \%$ reduction of amplitude of b-waves under both scotopic and photopic conditions - consistent with outer retina pathology involving both the rods and the cones (HP:0000548). At the same time, the child started showing unsteady gait (HP:0002317) and hand tremors (HP:0002378). Electroencephalogram, hearing, infectious biochemical profiles were normal, except for borderline elevation of acylcarnitine. Laboratory investigations for an autoimmune disorder were normal.

She was treated for 2 mo with biweekly pulse intravenous corticosteroids and immunoglobulin, with transient improvements in the nystagmus and gait abnormality but recurrence each time. By $17 \mathrm{mo}$, she began showing weakness of both upper extremities (HP:0003484), increased gait unsteadiness, a head tilt to the right side, and excessive drooling (HP:0002307). Physical examination showed no upper motor neuron signs in the lower extremities.

By 19 mo, she had shown no sustained response to steroid treatment. Given the rapidly progressing neurodegeneration and the continued possibility of an autoimmune disorder, treatment with cyclophosphamide-a chemotherapy agent-was considered as the next step. At the same time, an expedited genetic analysis was initiated given the possibility that a genetic diagnosis might change the treatment plan (approved by the Institutional Review Board of Duke University Medical Center).

\section{Genomic Analysis}

Interpretation of the patient's whole-exome sequence identified a compound heterozygous genotype in the solute carrier family 52 (riboflavin transporter), member 2 gene (SLC52A2). Loss-of-function mutations in SLC52A2 are associated with the autosomal recessive disorder Brown-Vialetto-Van Laere Syndrome 2 (BVVLS2), a progressive neurodegenerative disease that is a consequence of severe riboflavin deficiency. BVLS2 often results in respiratory failure and is associated with early mortality (Johnson et al. 2012; Foley et al. 2014).

The patient inherited a rare missense mutation $(0.015 \%$ ESP6503 minor allele frequency) from her heterozygous father NM_001253815.1:c.1016T>C p.(Leu339Pro) and a rare $(0.008 \%$ ESP6503 minor allele frequency) midprotein nonsense mutation from her 
Table 1. SLC52A2 (NM_001253815.1) variants

\begin{tabular}{|c|c|c|c|c|c|c|c|c|}
\hline $\begin{array}{l}\text { Chr:Position } \\
\text { GRCh37 } \\
\text { (hg19) }\end{array}$ & $\begin{array}{l}\text { HGVS } \\
\text { cDNA }\end{array}$ & $\begin{array}{l}\text { HGVS } \\
\text { protein }\end{array}$ & $\begin{array}{l}\text { Predicted } \\
\text { effect }\end{array}$ & $\mathrm{dbSNP} I D$ & Genotype & $\begin{array}{l}\text { Parent } \\
\text { of origin }\end{array}$ & Observed effect & $\begin{array}{l}\text { EVS } \\
\text { MAF (\%) }\end{array}$ \\
\hline $8: 145583960$ & c. $808 \mathrm{C}>\mathrm{T}$ & p. $\left(G \ln 270^{*}\right)$ & Stop gain & rs375088539 & Heterozygous & Mother & Degradation (Fig. 1) & 0.008 \\
\hline $8: 145584264$ & c. $1016 \mathrm{~T}>\mathrm{C}$ & p.(Leu339Pro) & Missense & rs148234606 & Heterozygous & Father & $\begin{array}{l}\text { Abolished riboflavin } \\
\text { uptake (Foley et al. } \\
\text { 2014) }\end{array}$ & 0.015 \\
\hline
\end{tabular}

Details about the two SLC52A2 loss-of-function variants identified in our patient. Chromosome and position coordinates based on GRCh37(hg19). Corresponding DNA and protein references are provided. EVS minor allele frequency (MAF) is based on up to 6503 samples. Both variants have been independently validated by a CLIA-certified laboratory.

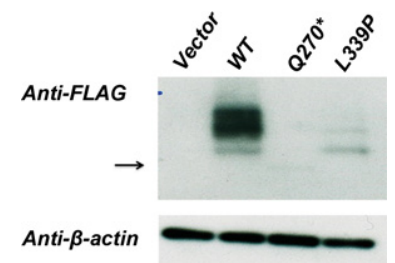

Figure 1. Western blot analysis using crude membrane fractions of HEK293 expressing the empty vector, WTSLC52A2-Flag (WT), Q270*SLC52A2-Flag (Q270X), and L339P-SLC52A2-Flag(L339P) variants. The blot was probed using anti-Flag and $\beta$-actin antibodies. The arrow highlights the location of the faint band of the truncated protein due to Q270* mutation at $\sim 30 \mathrm{kDa}$. The L339P mutation results in decreased protein expression. The functional effect of the L339P missense has been previously described (Foley et al. 2014). heterozygous mother NM_001253815.1:c.808C>T p.(Gln270*) (Table 1). The paternal missense variant (L339P) has been reported as pathogenic in multiple patients (Johnson et al. 2012; Foley et al. 2014), with in vitro assays showing abolishment of ${ }^{3} \mathrm{H}$-Riboflavin uptake for the mutant protein (Foley et al. 2014). The maternal nonsense variant has not been previously linked to BVVLS2. This Q270* nonsense variant, however, would be predicted to abolish protein function. Consistent with this expectation we showed that the nonsense variant results in a truncated protein that appears subject to degradation (Fig. 1). In summary, the patient has a compound heterozygous genotype made of two loss-of-function mutations in SLC52A2. Both variants were confirmed to be present in the patient by a CLIA-certified laboratory. Consistent with expectation of loss of function in a gene intolerant to functional variation (Petrovski et al. 2013), this genotype means that the patient in fact has BrownVialetto-Van Laere Syndrome 2, an autosomal recessive condition caused by loss-of-function mutations in both copies of SLC52A2.

\section{Immediate Treatment Outcomes}

In retrospect after the genetic diagnosis, the patient's presentation is considered a good match for the rare BVVLS2 diagnosis. Because of the urgent need to withdraw the scheduled precautionary chemotherapy for a possible autoimmune condition, and to introduce riboflavin, the whole-exome sequencing results and the correct diagnosis were communicated to the parents within $72 \mathrm{~h}$ of the genome interpretation. The same day, a $10 \mathrm{mg} / \mathrm{kg} / \mathrm{d}$ dose of riboflavin was initiated (Fig. 2) and titrated up to $70 \mathrm{mg} / \mathrm{kg} / \mathrm{d}$ over $2 \mathrm{mo}$. The child has tolerated the riboflavin therapy well. The parents reported a decrease in drooling within a week, followed by improved head tilt, an improved sense of well-being, and a renewed interest in play (Fig. 3). Two months after initiating therapy, the gait was noticed to be steadier and

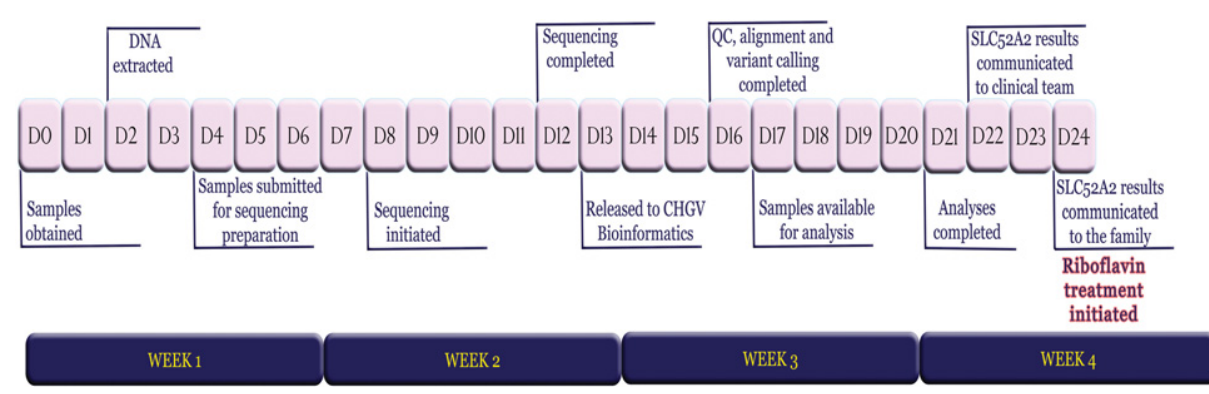

Figure 2. Timeline of events starting from the day of phlebotomy (D0) to the day that the SLC52A2 sequencing results were communicated to the family (D24). 
COLD SPRING HARBOR Molecular Case Studies
Exome sequencing guides appropriate patient care
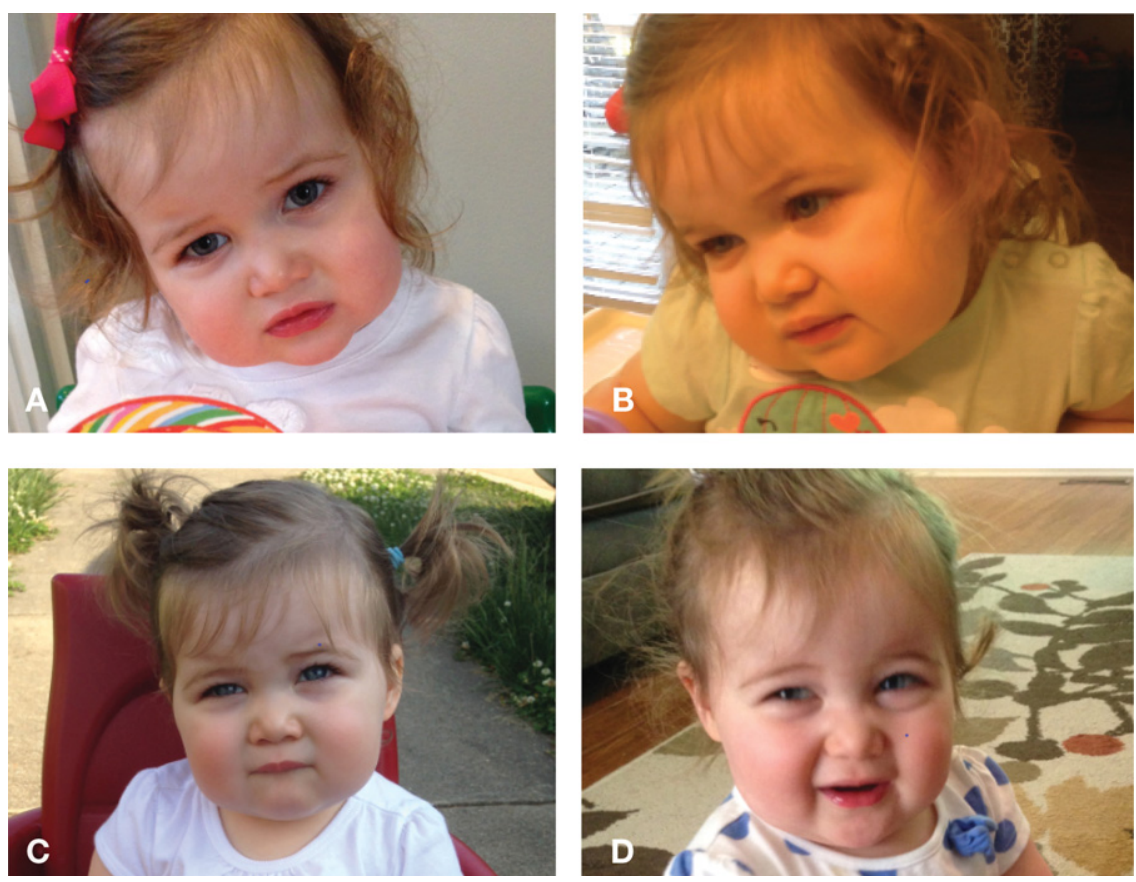

Figure 3. Head tilt, pre- and posttreatment with riboflavin supplementation. (A) Three weeks pretreatment; $(B)$ day 2 of treatment; (C) 3 wk posttreatment; (D) 4 wk posttreatment.

forearm strength had improved (power 3/5). Immediately upon initiating riboflavin therapy no new symptoms or further decline in any pretreatment symptoms were observed.

The patient's sister, a healthy appearing newborn female, had cord blood sent for genotyping of the familial SLC52A2 mutations. As a precautionary measure, she was initiated on $10 \mathrm{mg} / \mathrm{kg} / \mathrm{d}$ of riboflavin while awaiting the results. The genotyping results indicated that the newborn sister was not a carrier for either SLC52A2 loss-of-function allele and was appropriately weaned off the precautionary riboflavin supplementation.

\section{DISCUSSION}

The carrier frequency of SLC52A2 pathogenic mutations suggests approximately 1 in 4.3 million children will be affected globally (Exome Aggregation Consortium; exac.broadinstitute .org/). The population rate is consistent with the clinically cited rate of autosomal recessive BVVLS2 being less than 1 in a million. Interestingly, there have been fewer reported diagnoses in the United States than the 73 the carrier frequency would suggest. Such differences between the expected and reported numbers of cases could in part stem from early and aggressive, yet inaccurate, treatment compromising recognition of clinical features that might have enabled an eventually correct diagnosis. Consistent with this, in BVVLS2, riboflavin or FAD biochemical plasma screening generally return normal values, making BVVLS2 challenging to diagnose early. This expected rate of BVLS2 also suggests that there could be patients in the United States that are being mismanaged because of the failure to correctly diagnose their condition as BVVLS. We emphasize that sequencing and careful interpretation could result in a correct genetic diagnosis for such patients and ultimately facilitate changes in patient management.

To our knowledge, this is the first and earliest diagnosis of an isolated case of BVVLS2 through exome sequencing in the absence of clinical ascertainment for BVVLS, and it has 
resulted in the treatment of a patient at one of the youngest ages yet reported. This case adds to a rapidly growing number of examples in which exome sequencing provides a diagnosis that would not otherwise have been obtained and an immediate clinical benefit in the form of a genomic guided treatment (Choi et al. 2009; Bainbridge et al. 2011; Worthey et al. 2011; Bearden et al. 2014; Canna et al. 2014; Romberg et al. 2014). Our case also emphasizes that a timely genetic diagnosis using exome sequencing has avoided alternative toxic treatments and a patient management plan that would have been unlikely to help our patient's genetic condition. Finally, the expedited exome sequencing allowed us to provide prophylactic treatment to the at-risk newborn sibling while awaiting the results of her genotyping test. Such precautionary newborn screening highlights the possibility of implementing safe and effective presymptomatic treatment from birth.

It remains unclear what proportion of patients with serious genetic diseases would derive such clear clinical benefit from exome sequencing. Nevertheless, the accumulating evidence strongly suggests that this happens with sufficient regularity to warrant systematic application of exome sequencing, and eventually genome sequencing, in all unresolved childhood diseases where a genetic cause is suspected. Moreover, in a number of cases, including BVVLS2, the earlier that treatment is initiated, the better the prognosis. This creates a strong clinical imperative for applying exome sequencing as early as possible.

\section{MATERIALS AND METHODS}

\section{Sequencing and Analysis}

DNA was extracted from maternal, paternal, and proband blood samples and was exome sequenced on the HiSeq2500 using KAPA Biosystem's library preparation kitfollowed by wholeexome capture using Nimblegen SeqCap EZ V3.Orapid. Paired-end $2 \times 100$ read lengths were used for the exome capture sequencing. After processing the raw reads using a pipeline based on the GATK best practice protocol, the resulting alignments indicated that $96.2 \%$ of the $33,266,994$ consensus coding sequence CCDS (release 14) sites had $\geq 10$-fold coverage in all three family members. Individual-level coverage data indicate that all three family members had $100 \%$ of SLC52A2 covered with at least 10 -fold coverage (Table 2). The sequence data were analyzed using our established trio sequencing protocols (Zhu et al. 2015) that identify qualifying variants forming genotypes not observed in the parents or in an external database of 6503 controls of convenience provided by the Exome Sequencing Project (ESP6500SI) (NHLBI GO Exome Sequencing Project; evs.gs.washington.edu).

\section{Western Blot}

The human cDNA clone of SLC52A2 was ordered from PlasmID (Harvard Medical School, clone ID: HsCD00372773). The cDNA sequence was amplified using PrimeSTAR GXL

Table 2. Sequencing coverage

\begin{tabular}{|c|c|c|c|c|c|c|}
\hline Sample & $\begin{array}{l}\text { Percentage of } \\
\text { reads aligned }\end{array}$ & $\begin{array}{l}\text { Average } \\
\text { read } \\
\text { coverage }\end{array}$ & $\begin{array}{l}\text { Number of CCDS } \\
\text { r14 sites with } \geq 10- \\
\text { fold coverage }\end{array}$ & $\begin{array}{l}\text { Percentage of CCDS } \\
\text { r14 with } \geq 10 \text {-fold } \\
\text { coverage }\end{array}$ & $\begin{array}{l}\text { Percentage of CCDS } \\
\text { r14 with } \geq 20 \text {-fold } \\
\text { coverage }\end{array}$ & $\begin{array}{l}\text { Percentage of SLC52A2 } \\
\text { sites (1354 bases) with } \\
\geq 10 \text {-fold coverage }\end{array}$ \\
\hline Proband & 99.51 & 63.91 & $32,282,510$ & $97.04 \%$ & $92.43 \%$ & 100 \\
\hline Mother & 99.47 & 74.84 & $32,491,343$ & $97.67 \%$ & $94.64 \%$ & 100 \\
\hline Father & 99.62 & 93.58 & $32,658,451$ & $98.17 \%$ & $95.93 \%$ & 100 \\
\hline
\end{tabular}

Sequencing coverage information for each of the three exome-sequenced family members. The $33.3 \mathrm{Mbp}$ of consensus coding sequence (CCDS release 14 ) is used as the reference for protein-coding sequence coverage-this includes a 2-bp extension at both ends of exons to include putative canonical splice variants. 
Table 3. Primer sequences used to create constructs for immunoblotting

\begin{tabular}{ll}
\hline Primer name & Primer sequence \\
\hline SLC52A2-HindllI-F & ttAAGCTTGCAGCACCCACGCCCGCCCG \\
SLC52A2-BamH1 & gtGGATCCTCAGGAGTCACAGGGGTCTG \\
SLC52A2-MutaF-O270* & TAGCTTCTATCAGCCCGCAG \\
SLC52A2-MutaR-O270* & ATAGGCCTTAGGGTCTGGAC \\
SLC52A2-MutaF-L339P & CGGGCGGCCTCTCTCTGCT \\
SLC52A2-MutaR-L339P & GCCCTGCCAAGGACCTGCA \\
\hline
\end{tabular}

DNA Polymerase (Takara, ID: R050A) and primers (Table 3) that introduced a Hindlll restriction site $3^{\prime}$ of the sequence and a BamH1 site $5^{\prime}$ of the sequence. The linear CDNA and the p3XFlag-CMV 7.1 vector (Sigma-Aldrich, ID: E7533) were then digested with HindIII and BamH1 and ligated together using T4-ligase (New England Biolabs, ID: M0202S). The wild-type construct, WT-SLC52A2-Flag, was used to create Q270*-SLC52A2-Flag and L339P-SLC52A2-Flag by site-directed mutagenesis introducing the C>T (Q270*) and T>C (L339P) mutations (Table 3). Each construct was sequenced by Sanger to verify mutation and full cDNA sequence. HEK293 cells were transfected in triplicate using each of the listed constructs, and lysates were collected $48 \mathrm{~h}$ after transfection using RIPA buffer (SigmaAldrich, ID: R0278) and $1 \times$ protease inhibitor (Thermo Scientific, ID:88266). Protein concentrations were determined using the Pierce BCA protein assay kit (Thermo Scientific, ID: 23227). Of note, $2.5 \mu \mathrm{g}$ of lysate was subjected to SDS-PAGE gel and transferred to a polyvinylidene difluoride membrane (Millipore, ID: ISEQ15150). The membrane was incubated with anti-Flag antibody (1:2000; Sigma-Aldrich, ID: F3165) or anti- $\beta$-actin (1:5000; Santa Cruz, ID: sc-47778). Proteins were visualized with the ECL Plus Western Blotting Detection System (GE Healthcare, ID: RPN2232).

\section{ADDITIONAL INFORMATION}

\section{Ethics Statement}

The Institutional Review Board of Duke University Medical Center approved this research protocol (Pro00032301). Written informed consent was received from both parents.

\section{Data Deposition and Access}

Our patient consent does not permit patient sequence data to be uploaded to a data repository. The NM_001253815.1:p.(Leu339Pro) missense variant can be accessed through dbSNP rs148234606, and ClinVar SCV000221313. The NM_001253815.1:p.(Gln270*) nonsense variant can be accessed through dbSNP rs375088539, and ClinVar SCV000218457.

\section{Acknowledgments}

We thank the family for their enthusiasm to share their experience with the larger community. We thank members of the Institute for Genomic Medicine, formerly Center for Human Genome Variation, (Joshua Bridgers, Kenneth Cronin [former], Sally Gewalt, Jonathan Keebler [former], Zhong Ren, and Quanli Wang) for their ongoing commitment that enables this work. We would like to thank Angelica Struve and Tom Maniatis from the Maniatis Laboratory for their help and advice. We thank Dr. A. Reghan Foley of the Neuromuscular and Neurogenetic Disorders of Childhood Section, National Institute of Neurological Disorders and Stroke, National Institutes of Health, for her recommendations on the

Received January 23, 2015; accepted in revised form April 2, 2015. 
riboflavin dosing regimen and clinical surveillance while on this regimen, based on findings from studying an international cohort of BVVLS2 patients. We also thank the NHLBI GO Exome Sequencing Project and its ongoing studies that produced and provided exome variant calls for comparison: the Lung GO Sequencing Project (HL-102923), the WHI Sequencing Project (HL-102924), the Broad GO Sequencing Project (HL-102925), the Seattle GO Sequencing Project (HL-102926), and the Heart GO Sequencing Project (HL103010). S.P. is a National Health and Medical Research Council (NHMRC) CJ Martin Fellow. The authors wish to thank the Exome Aggregation Consortium and the groups that provided exome variant data for comparison. A full list of contributing groups can be found at http://exac.broadinstitute.org/about.

\section{Author Contributions}

Patient recruitment and phenotyping: V.S., K.S., R.C., L.C., R.K., M.A.E-D., Y-H.J., and M.A.M. Sequence data analysis and interpretation: S.P. and D.B.G. Functional evaluation of variant: K.M.M., R.S.D., B.K., and S.P. Writing of manuscript: S.P., V.S., and D.B.G. All authors contributed to reviewing the final draft.

\section{REFERENCES}

Bainbridge MN, Wiszniewski W, Murdock DR, Friedman J, Gonzaga-Jauregui C, Newsham I, Reid JG, Fink JK, Morgan MB, Gingras MC, et al. 2011. Whole-genome sequencing for optimized patient management. Sci Transl Med 3: 87re3.

Bearden D, Strong A, Ehnot J, DiGiovine M, Dlugos D, Goldberg EM. 2014. Targeted treatment of migrating partial seizures of infancy with quinidine. Ann Neurol 76: 457-461.

Bosch AM, Stroek K, Abeling NG, Waterham HR, ljlst L, Wanders RJ. 2012. The Brown-Vialetto-Van Laere and Fazio Londe syndrome revisited: natural history, genetics, treatment and future perspectives. Orphanet J Rare Dis 7: 83.

Canna SW, de Jesus AA, Gouni S, Brooks SR, Marrero B, Liu Y, DiMattia MA, Zaal KJ, Sanchez GA, Kim H, et al. 2014. An activating NLRC4 inflammasome mutation causes autoinflammation with recurrent macrophage activation syndrome. Nat Genet 46: 1140-1146.

Choi M, Scholl UI, Ji W, Liu T, Tikhonova IR, Zumbo P, Nayir A, Bakkaloglu A, Ozen S, Sanjad S, et al. 2009. Genetic diagnosis by whole exome capture and massively parallel DNA sequencing. Proc Natl Acad Sci 106: 19096-19101.

Foley AR, Menezes MP, Pandraud A, Gonzalez MA, Al-Odaib A, Abrams AJ, Sugano K, Yonezawa A, Manzur AY, Burns J, et al. 2014. Treatable childhood neuronopathy caused by mutations in riboflavin transporter RFVT2. Brain 137: 44-56.

Haack TB, Makowski C, Yao Y, Graf E, Hempel M, Wieland T, Tauer U, Ahting U, Mayr JA, Freisinger P, et al. 2012. Impaired riboflavin transport due to missense mutations in SLC52A2 causes Brown-Vialetto-Van Laere syndrome. J Inherit Metab Dis 35: 943-948.

Johnson JO, Gibbs JR, Megarbane A, Urtizberea JA, Hernandez DG, Foley AR, Arepalli S, Pandraud A, SimónSánchez J, Clayton $P$, et al. 2012. Exome sequencing reveals riboflavin transporter mutations as a cause of motor neuron disease. Brain 135: 2875-2882.

Köhler S, Doelken SC, Mungall CJ, Bauer S, Firth HV, Bailleul-Forestier I, Black GC, Brown DL, Brudno M, Campbell J, et al. 2014. The Human Phenotype Ontology project: linking molecular biology and disease through phenotype data. Nucleic Acids Res 42: D966-D974.

Petrovski S, Wang Q, Heinzen EL, Allen AS, Goldstein DB. 2013. Genic intolerance to functional variation and the interpretation of personal genomes. PLoS Genet 9: e1003709.

Romberg N, Al Moussawi K, Nelson-Williams C, Stiegler AL, Loring E, Choi M, Overton J, Meffre E, Khokha MK, Huttner AJ, et al. 2014. Mutation of NLRC4 causes a syndrome of enterocolitis and autoinflammation. Nat Genet 46: 1135-1139.

Worthey EA, Mayer AN, Syverson GD, Helbling D, Bonacci BB, Decker B, Serpe JM, Dasu T, Tschannen MR, Veith RL, et al. 2011. Making a definitive diagnosis: successful clinical application of whole exome sequencing in a child with intractable inflammatory bowel disease. Genet Med 13: 255-262.

Zhu X, Petrovski S, Xie P, Ruzzo EK, Lu YF, McSweeney KM, Ben-Zeev B, Nissenkorn A, Anikster Y, Oz-Levi D, et al. 2015. Whole-exome sequencing in undiagnosed genetic diseases: interpreting 119 trios. Genet Med doi: 10.1038/gim.2014.191. 


\section{COLD SPRING HARBOR Molecular Case Studies}

\section{Exome sequencing results in successful riboflavin treatment of a rapidly progressive neurological condition}

Slavé Petrovski, Vandana Shashi, Steven Petrou, et al.

Cold Spring Harb Mol Case Stud 2015, 1: a000257

Access the most recent version at doi: $10.1101 / \mathrm{mcs} .0000257$

References

License

Email Alerting Service
This article cites 13 articles, 2 of which can be accessed free at: http://molecularcasestudies.cshlp.org/content/1/1/a000257.full.html\#ref-list-1

This article is distributed under the terms of the Creative Commons Attribution-NonCommercial License, which permits reuse and redistribution, except for commercial purposes, provided that the original author and source are credited.

Receive free email alerts when new articles cite this article - sign up in the box at the top right corner of the article or click here. 